\title{
LIMITS OF PURE STATES, II
}

\author{
by R. J. ARCHBOLD and A. M. ZAKI
}

(Received 18th June 1990)

We answer a question raised in an earlier paper concerning the pure state space of a separable $C^{*}$-algebra.

1980 Mathematics subject classification (1985 Revision): $46 \mathrm{~L} 30$

Let $A$ be a unital separable $C^{*}$-algebra with state space $S(A)$. Let $P(A)$ denote the set of pure states of $A$ and let $F(A)$ denote the set of factorial states. For $\phi \in S(A)$ let $\pi_{\phi}$ be the associated Gelfand-Naimark-Segal representation of $A$. It was shown in Archbold [1] that the mapping $\theta$ defined by $\theta(\phi)=\operatorname{ker} \pi_{\phi}$ is a continuous, open surjection from $\overline{F(\mathrm{~A})}$ (the weak*-closure of $F(A)$ ) onto $\operatorname{Primal}^{*}(A)$ (the set of proper primal ideals of $A$ ). Furthermore, the restriction $\theta_{0}$ of $\theta$ to the pure state space $P(A)$ is also surjective (Archbold [2]).

Question 2 in Archbold [2] asks whether $\theta_{0}$ is open. In certain special cases the answer is affirmative. For example, if $A$ is antiliminal than $\overline{P(\mathrm{~A})}=\overline{F(A)}$ (Batty and Archbold [4]) and so $\theta_{0}=\theta$. Furthermore, we show that $\theta_{0}$ is always "almost open" in the sense that the image of any non-empty open set has dense interior. However, $\theta_{0}$ can fail to be open and we give an example in which $A$ is liminal and the primitive ideal space $\operatorname{Prim}(A)$ is Hausdorff.

We begin by recalling from Archbold and Batty [3] that a (closed two-sided) ideal $J$ of $A$ is said to be primal if whenever $n \geqq 2$ and $J_{1}, J_{2}, \ldots, J_{n}$ are ideals of $A$ such that $J_{1} J_{2} \ldots J_{n}=\{0\}$ then $J_{i} \subseteq J$ for at least one value of $i$. In this paper we shall be concerned with the weak topology $\tau_{w}$ on $\operatorname{Primal}^{\prime}(A)$ (see Archbold [1]). A base is given by the family of sets of the form

$$
U(F)=\left\{I \in \operatorname{Primal}^{\prime}(A): J \nsubseteq I \text { for all } J \in F\right\}
$$

where $F$ is a finite set (possibly empty) of ideals of $A$. When restricted to Prim $(A)$, $\tau_{w}$ coincides with the Jacobson topology. If Prim $(A)$ is Hausdorff then $\operatorname{Primal}^{\prime}(A)=\operatorname{Prim}(A)$ (see Archbold and Batty [3, p. 63]).

Theorem. Let $A$ be a unital separable $C^{*}$-algebra and let $\theta_{0}: \overline{P(A)} \rightarrow \operatorname{Primal}^{\prime}(A)$ be defined by

$$
\theta_{0}(\phi)=\operatorname{ker} \pi_{\phi} \quad(\phi \in \overline{P(A)})
$$

Let $U$ be any non-empty open subset of $\overline{P(A)}$. Then the interior of $\theta_{0}(U)$ is dense in $\theta_{0}(U)$. 
Proof. Let $W=U \cap P(A)$, a non-empty open subset of $P(A)$. By Pedersen [6, 4.3.3], $\theta_{0}(W)$ is a non-empty open subset of Prim $(A)$. Hence there exists a non-zero ideal $J$ of $A$ such that

\section{Define}

$$
\theta_{0}(W)=\{P \in \operatorname{Prim}(A): P \notin J\}
$$

$$
V=\left\{I \in \operatorname{Primal}^{\prime}(A): I \notin J\right\},
$$

a $\tau_{w}$-open subset of $\operatorname{Primal}^{\prime}(A)$. Since $W$ is dense in $U$ and $\theta_{0}$ is continuous (see Archbold [1, Section 2]), we have

$$
\bar{V} \supseteq \overline{\theta_{0}(W)} \supseteq \theta_{0}(\bar{W}) \supseteq \theta_{0}(U)
$$

(where the bars denote closures in the appropriate topologies). It remains only to show that $V \subseteq \theta_{0}(U)$.

Let $I \in V$. Since $I \notin J$ there exists a primitive ideal $P_{1}$ of $A$ such that $P_{1} \supseteq I$ and $P_{1} \neq J$ (Dixmier $[5,2.9 .7(\mathrm{ii})]$ ). Hence $P_{1} \in \theta_{0}(W)$ and so there exists $\phi_{1} \in W$ such that $P_{1}=\operatorname{ker} \pi_{\phi_{1}}$. Since $A$ is separable there is a countable family $P_{1}, P_{2}, \ldots$ of distinct elements of Prim $A$ whose intersection is $I$ (Pedersen [6, 4.3.4]). We shall assume that this family is infinite (in the finite case, a similar but easier argument applies).

For $i \geqq 2$ let $\phi_{i}$ be a pure state such that $P_{i}=\operatorname{ker} \pi_{\phi_{i}}$. For $n \geqq 1$ let

$$
\psi_{n}=\frac{n-1}{n} \phi_{1}+\frac{1}{n} \sum_{i=2}^{\infty} 2^{-i+1} \phi_{i}
$$

Since $I$ is primal, it follows from Archbold and Batty [3, Proposition 3.1] that there is a net $\left(\pi_{\alpha}\right)$ of irreducible representations of $A$ such that $\pi_{\alpha} \rightarrow \pi_{\phi_{i}}$ for each $i \geqq 1$. By Archbold [2, Theorem $2((\mathrm{ii}) \Rightarrow(\mathrm{iii}))], \psi_{n} \in \overline{P(A)}$. Since $\left\|\psi_{n}-\phi_{1}\right\| \leqq 2 / n$ and $\phi_{1} \in U$, there exists $N$ such that $\psi_{N} \in U$. However,

$$
\operatorname{ker} \pi_{\psi_{N}}=\bigcap_{i=1}^{\infty} \operatorname{ker} \pi_{\phi_{i}}=\bigcap_{i=1}^{\infty} P_{i}=I
$$

Hence $I \in \theta_{0}(U)$ as required.

We now give an example in which the map $\theta_{0}$ is not open. Let $M_{n}(\mathbf{C})$ denote the $C^{*}$-algebra of all $n \times n$ complex matrices. Let $B$ be the $C^{*}$-subalgebra of $M_{6}(\mathrm{C})$ consisting of all matrices of the form $\left[\begin{array}{ll}S & 0 \\ 0 & T\end{array}\right]$ where $S \in M_{4}(C)$ and $T \in M_{2}(C)$. We define $A$ to be the $C^{*}$-algebra of all sequences $x=\left(x_{n}\right)_{n \geqq 1}$, where $x_{n}=\left[\begin{array}{cc}S_{n}(x) & 0 \\ 0 & T_{n}(x)\end{array}\right] \in B$, which are convergent to a matrix of the form 


$$
\left[\begin{array}{ccc}
T(x) & 0 & 0 \\
0 & T(x) & 0 \\
0 & 0 & T(x)
\end{array}\right]
$$

where $T(x) \in M_{2}(C)$. The algebraic operations in $A$ are defined pointwise and the norm is the supremum norm.

For each $n \geqq 1$ there are irreducible representations $\pi_{n}$ and $\sigma_{n}$ of $A$ given by $\pi_{n}(x)=S_{n}(x)$ and $\sigma_{n}(x)=T_{n}(x)$ for $x \in A$. The only other irreducible representation is the representation $\sigma_{\infty}$ given by $\sigma_{\infty}(x)=T(x)$ for $x \in A$. Hence Prim $(A)$ consists of the ideals $P_{n}, Q_{n}(n \geqq 1)$ and $Q_{\infty}$ where $P_{n}=\operatorname{ker} \pi_{n}, Q_{n}=\operatorname{ker} \sigma_{n}$ and

$$
Q_{\infty}=\operatorname{ker} \sigma_{\infty}=\left\{x \in A: x_{n} \rightarrow 0 \text { as } n \rightarrow \infty\right\} \text {. }
$$

Each $P_{n}$ and each $Q_{n}$ is an isolated point in $\operatorname{Prim}(A)$. However $P_{n} \rightarrow Q_{\infty}$ and $Q_{n} \rightarrow Q_{\infty}$ as $n \rightarrow \infty$. In particular, Prim $(A)$ is Hausdorff and so $\operatorname{Primal}^{\prime}(A)=\operatorname{Prim}(A)$. We note also that $A$ is unital, separable and liminal.

We define $\phi \in S(A)$ by

$$
\phi(x)=\operatorname{tr}\left(\sigma_{\infty}(x)\right)=\operatorname{tr}(T(x)) \quad(x \in A)
$$

where $\operatorname{tr}$ is the (unique) tracial state of $M_{2}(\mathrm{C})$. We shall show that $\phi \in \overline{P(A)}$ and that there exists an open neighbourhood $U$ of $\phi$ in $\overline{P(A)}$ such that $\theta_{0}(U)$ is not a neighbourhood of $\theta_{0}(\phi)\left(=Q_{\infty}\right)$. To see that $\phi \in \overline{P(A)}$, let $\xi=2^{-1 / 2}(1,0,0,1) \in \mathbf{C}^{4}$ and define $\phi_{n} \in P(A)$ by

$$
\phi_{n}(x)=\left\langle\pi_{n}(x) \xi, \xi\right\rangle \quad(x \in A)
$$

Then

$$
\begin{aligned}
\phi_{n}(x)= & \frac{1}{2}\left[\left(S_{n}(x)\right)_{11}+\left(S_{n}(x)\right)_{14}+\left(S_{n}(x)\right)_{41}+\left(S_{n}(x)\right)_{44}\right] \\
& \rightarrow \frac{1}{2}\left[T(x)_{11}+0+0+T(x)_{22}\right]
\end{aligned}
$$

as $n \rightarrow \infty$. Thus $\phi_{n} \rightarrow \phi\left(\right.$ weak $\left.^{*}\right)$ and so $\phi \in \overline{P(A)}$.

Now let $U$ be the complement in $\overline{P(A)}$ of the weak*-closure of the set

$$
W=\left\{\psi \in \overline{P(A)}: \operatorname{ker} \pi_{\psi}=Q_{n} \text { for some } n\right\} .
$$

It suffices to show that $\phi \in U$ for then $Q_{\infty}=\theta_{0}(\phi) \in \theta_{0}(U)$ but $\theta_{0}(U)$ is not a neighbourhood of $Q_{\infty}$ since $Q_{n} \notin \theta_{0}(U)$ for each $n$.

We show first of all that $W \subseteq P(A)$. So let $\psi \in \overline{P(A)}$ with ker $\pi_{\psi}=Q_{n}$ for some $n$. There exists a net $\left(\psi_{\alpha}\right)$ in $P(A)$ such that $\psi_{a} \rightarrow \psi$. Since $\theta_{0}$ is continuous, $\operatorname{ker} \pi_{\psi_{\alpha}} \rightarrow Q_{n}$ in Prim $(A)$. Hence ker $\pi_{\psi_{z}}$ is eventually equal to $Q_{n}$. Regarding $\psi$ as a state of $A / Q_{n}\left(\cong M_{2}(\mathrm{C})\right)$ in the usual way, we obtain that 


$$
\psi \in \overline{P\left(A / Q_{n}\right)}=P\left(A / Q_{n}\right)
$$

as required.

Let us suppose that $\phi \notin U$. Then there exists a net $\left(\phi_{\alpha}\right)$ in $W$ such that $\phi_{\alpha} \rightarrow \phi$. Let $Q_{n_{\alpha}}=\operatorname{ker} \pi_{\phi_{\alpha}}$. Since $\phi_{\alpha}$ is pure, there exists a unit vector $\xi_{\alpha} \in C^{2}$ such that

$$
\phi_{\alpha}(x)=\left\langle\sigma_{n_{\alpha}}(x) \xi_{\alpha}, \xi_{\alpha}\right\rangle \quad(x \in A)
$$

By passing to a subnet if necessary we may suppose that the net $\left(\xi_{\alpha}\right)$ is convergent to some unit vector $\xi \in \mathbf{C}^{2}$. Since $\phi_{\alpha} \rightarrow \phi$ and $\theta_{0}$ is continuous, $Q_{n_{\alpha}} \rightarrow Q_{\infty}$ in $\operatorname{Prim}(A)$.

Let $x \in A$ and $\varepsilon>0$. There exists $N \geqq 1$ such that $\left\|T_{n}(x)-T(x)\right\|<\varepsilon / 2$ for all $n \geqq N$. Since $Q_{n_{a}} \rightarrow Q_{\infty}$, there exists $\alpha_{0}$ such that $n_{a} \geqq N$ for all $\alpha \geqq \alpha_{0}$. By increasing $\alpha_{0}$ if necessary, we may assume that

$$
\left\|\xi_{\alpha}-\xi\right\|<\varepsilon(1+4\|T(x)\|)^{-1}
$$

for all $\alpha \geqq \alpha_{0}$. Then for $\alpha \geqq \alpha_{0}$ we have

$$
\begin{aligned}
\left.\mid \phi_{\alpha}(x)-\langle T(x) \xi, \xi\rangle\right) \mid & =\left|\left\langle T_{n_{\alpha}}(x) \xi_{\alpha}, \xi_{\alpha}\right\rangle-\langle T(x) \xi, \xi\rangle\right| \\
& <\left|\left\langle T(x) \xi_{\alpha}, \xi_{\alpha}\right\rangle-\langle T(x) \xi, \xi\rangle\right|+\frac{\varepsilon}{2} \\
& \leqq\left\|T(x)\left(\xi_{\alpha}-\xi\right)\right\|\left\|\xi_{\alpha}\right\|+\|T(x) \xi\|\left\|\xi_{\alpha}-\xi\right\|+\frac{\varepsilon}{2} \\
& \leqq \varepsilon .
\end{aligned}
$$

Hence $\phi(x)=\lim \phi_{a}(x)=\langle T(x) \xi, \xi\rangle$. This shows that $\phi$ is pure, contradicting the fact that $\phi$ is defined to be the average of two distinct pure states. This contradiction shows that $\phi \in U$, as required.

We note that in this example

$$
\theta_{0}(U)=\left\{P_{n}: n \geqq 1\right\} \cup\left\{Q_{\infty}\right\}
$$

The interior of $\theta_{0}(U)$ is $\left\{P_{n}: n \geqq 1\right\}$. This is dense in $\theta_{0}(U)$ (since $P_{n} \rightarrow Q_{\infty}$ as $n \rightarrow \infty$ ) as predicted by the theorem.

Acknowledgement. Part of this work was done while the first-named author was visiting the University of Aswan. The support of the British Council and the hospitality of the host institution are gratefully acknowledged. 


\section{REFERENCES}

1. R. J. ARChrold, Topologies for primal ideals, J. London Math. Soc. (2) 36 (1987), 524-542.

2. R. J. ARchrold, Limits of pure states, Proc. Edinbrugh Math. Soc. 32 (1989), 249-254.

3. R. J. ARChBold and C. J. K. BATTY, On factorial states of operator algebras, III, J. Operator Theory 15 (1986), 53-81.

4. C. J. K. Batty and R. J. ARChBold, On factorial states of operator algebras, II, J. Operator Theory 13 (1985), 131-142.

5. J. Dixmier, Les $C^{*}$-algèbres et leurs représentations (2nd edition, Gauthier-Villars, Paris, 1969).

6. G. K. Pedersen, C*-Algebras and their Automorphism Groups (Academic Press, London, 1979).

Department of Mathematical Sciences

The Edward Wright Building

Dunbar Street

Aberdeen AB9 2TY
Department of Mathematics

FaCULTY OF SCIENCE

Aswan

EGYPT 